\title{
Accidente vascular encefálico. Presentación clínica y factores de riesgo
}

\author{
MARTA HERNÁNDEZ CH. ${ }^{1}$, CATALINA SAMSÓ Z. ${ }^{2}$, RAÚL ESCOBAR H. ${ }^{1}$, TOMÁS MESA L. ${ }^{1}$ \\ 1. Neuropediatra, Facultad de Medicina, División de Pediatría, Sección Neurología Pediátrica. \\ Pontificia Universidad Católica de Chile. \\ 2. Becada neuropediatría. Facultad de Medicina, División de Pediatría, Sección Neurología Pediátrica. \\ Pontificia Universidad Católica de Chile.
}

\begin{abstract}
Clinical presentation and risk factors of cerebrovascular accident

Introduction: Acute focal brain dysfunction in adults serves to diagnose and to provide early rescue of the brain tissue. In children, the clinical differences of this condition compared to adults results in delayed diagnosis and treatment. Objective: To describe the clinical presentation and risk factors of CVA in 156 children and related to age and type of CVA. Patients and Method: A CVA registry of newborn to 18 years old was started in January 2003 were analized. Diagnosis was confirmed by brain imaging. Results: The median of age was 4.9 month (33\% was newborn, $46 \%<6$ years old, $21 \%>6$ years old); $64 \%$ were males. Arterial ischemic stroke represented $85 \%$, the rest were cerebral sinovenous thrombosis. In the clinical manifestations the diffuse signs were more frequently seen $(67 \%)$ in children $<6$ years of age ( $\mathrm{p} 0.001)$. Seizures were observed in $61 \%$ in children $<6$ years old $(\mathrm{p}=0.03), 33 \%$ presented focal signs. The most common risk factors were acute systemic diseases and heart disease. $14.7 \%$ of the patients did not present risk factors. Conclusions: Acute focus signals of CVA in children have no diagnostic value, unlike adults; however absence doesn't exclude focal ischemic vascular disease. The acute systemic disease was the most common risk factor for stroke.

(Key words: Acute Brain Dysfunction, pediatrics, ischemic stroke, risk factors, venous sinus thrombosis). Rev Chil Pediatr 2013; 84 (4): 396-402
\end{abstract}

\section{RESUMEN}

Introducción: La alteración focal aguda de la función cerebral en el adulto permite diagnosticar un accidente vascular encefálico (AVE) e iniciar precozmente el rescate de tejido cerebral. En los niños, las diferencias clínicas de esta patología en relación al adulto generan atraso, subdiagnóstico e impiden el tratamiento en la etapa aguda. Objetivo: Describir la presentación clínica y factores de riesgo del AVE isquémico en 156 niños, comparar la presentación clínica según edad y tipo de AVE. Pacientes y Método: Se analiza un registro

Recibido el 16 de noviembre de 2012, devuelto para corregir el 20 de febrero de 2013, segunda versión 23 de marzo de 2013 , aceptado para publicación el 08 de abril de 2013.

Este trabajo cumple con los requisitos sobre consentimiento /asentimiento informado, comité de ética, financiamiento, estudios animales y sobre la ausencia de conflictos de intereses según corresponda.

Correspondencia a:

Dra Marta Hernández Ch.

E-mail: mhernand@med.puc.cl 
de AVE isquémico, confirmado con imágenes cerebrales, en población menor de 18 años, iniciado en Enero 2003. Resultados: La mediana de edad: 4,9 meses (33\% recién nacidos, $46 \%$ menores 6 años y $21 \%$ mayor de 6 años), 64\% fueron hombres. Los infartos arteriales correspondieron al $85 \%$ y el resto fueron trombosis de senos venosos. Predominaron los signos difusos $(67 \%)$ en menores de 6 años $(p=0,001)$. Las convulsiones fueron observadas en el $61 \%$ de los niños menores de 6 años $(p=0,03)$, el $33 \%$ presentó signos focales. Los factores de riesgo más frecuentes fueron patologías sistémicas agudas y cardiopatías. En $14,7 \%$ no se encontraron factores de riesgo. Conclusiones: La búsqueda de signos focales agudos de AVE no tiene utilidad diagnóstica en el niño, a diferencia de los adultos, pues su ausencia no excluye patología vascular isquémica focal. La patología sistémica aguda fue el factor de riesgo más frecuente para AVE.

(Palabras clave: Disfunción Cerebral Aguda, pediatría, AVE isquémico, factores de riesgo, trombosis de senos venosos).

Rev Chil Pediatr 2013; 84 (4): 396-402

\section{Introducción}

La OMS define el accidente vascular encefálico (AVE) como "el inicio abrupto de signos neurológicos focales de origen vascular que dura más de $24 \mathrm{~h}$ "1. Esto ha llevado a que la población consulte y el equipo de salud diagnostique precozmente el evento, permitiendo rescatar el tejido afectado bajo el lema "tiempo es cerebro" 2,3 . En la población infantil, el AVE es una patología grave pero subdiagnosticada. Está entre las 10 primeras causas de mortalidad infantil y se describe hasta un $60 \%$ de secuelas motoras y cognitivas que ejercen su impacto durante muchas décadas ${ }^{4-6}$.

Sin embargo, es difícil diagnosticar AVE en la población pediátrica porque hay poca sospecha clínica, la presentación es variada e inespecífica, hay una amplia gama de diagnóstico diferencial y múltiples factores de riesgo. Todos estas causas llevan a retraso diagnóstico y subdiagnóstico en más de la mitad de los casos, lo que priva al paciente de los beneficios de una intervención precoz ${ }^{7-10}$. El tiempo entre el primer síntoma y el diagnóstico, en centros terciarios de USA y Canadá, es de $42 \mathrm{~h}^{11-13}$.

Los factores de riesgo del adulto como la hipertensión, hipercolesterolemia, arteriosclerosis, diabetes, tabaquismo y obesidad, son muy infrecuentes en niños. Entre los factores de riesgo más frecuentes en la edad pediátrica están las cardiopatías (congénitas y adquiridas), infecciones, enfermedades metabólicas, traumas de cráneo y cuello, vasculitis, trombofilias (hereditarias o adquiridas), y enfer- medades crónicas (síndrome nefrótico, colitis ulcerosa, entre otras). A pesar de lo anterior, hasta en un 30\% de los AVE infantiles no se encuentra causa y en un $40 \%$ hay más de dos factores de riesgo ${ }^{14-17}$.

La aparición de signos neurológicos agudos en niños con factores de riesgo conocidos debe hacer sospechar un AVE, aún en ausencia de focalización neurológica. En lactantes, la presentación usual del AVE es convulsiones, compromiso de conciencia y compromiso del estado general, que también puede ser la presentación de una sepsis, estado gripal u otro trastorno agudo del lactante. Los signos focales como hemiparesia o déficit visual agudo pueden estar ausentes, pueden aparecer transitoriamente, aun en presencia de una lesión permanente o ser difícilmente reconocibles por el pediatra general ${ }^{12,18-21}$.

El objetivo de este reporte es describir la presentación clínica de 156 niños con AVE isquémico y comparar los síntomas en relación a la edad y subtipo de AVE.

\section{Pacientes y Método}

Se realizó un análisis retrospectivo de una cohorte de niños con AVE isquémico, reclutado desde el año de 2003 a febrero de 2012 en un registro de AVE. Los pacientes ingresaron a un centro terciario o fueron controlados antes de las dos semanas del AVE en la Pontificia Universidad Católica de Chile (PUC). Este estudio fue aprobado por el Comité de Ética de la PUC. 
Criterios de inclusión: Recién nacidos de término (37 semanas), niños y adolescentes hasta 18 años de edad al momento del AVE; neuroimágenes que confirman el AVE isquémico (resonancia magnética cerebral o tomografía computada), diagnóstico reciente (no más de tres semanas del evento). Criterios de exclusión: crisis isquémicas transitorias y datos incompletos.

La recolección de datos se realizó en una ficha clínica diseñada que registra datos del cuadro clínico inicial, imágenes cerebrales, tipo de AVE, exámenes realizados, evolución al alta y controles posteriores.

La presentación clínica de los pacientes se dividió en síntomas agudos focales, difusos y convulsiones. Los síntomas focales se definieron como aquellos que permiten localizar la zona cerebral afectada (Ej. una hemiparesia izquierda evoca una lesión en la zona motora o cápsula interna derecha). Los síntomas difusos incluyeron cefalea, náuseas, vómitos y compromiso de conciencia; estos sugieren un compromiso extenso del cerebro pero no permiten localizar la lesión. Una convulsión fue definida como una descarga neuronal paroxística, que altera en forma transitoria la función cerebral.

Los factores de riesgo se clasificaron en enfermedades agudas sistémicas (fiebre, shock, sepsis, deshidratación, acidosis) cardiopatías (congénitas y adquiridas), enfermedades crónicas sistémicas y enfermedades crónicas de cabeza-cuello (síndrome nefrótico, cánceres, colitis ulcerosa, trombofilias, Sturge Weber, PHACES etc.), vasculopatías o arteriopatías (moyamoya, disección arterial, vasculitis etc.) y enfermedades agudas de cabeza y cuello (meningitis, faringitis, cirugía reciente, infección senos paranasales), según el International Pediatric stroke study ${ }^{22}$. Las categorías de factores de riesgo no son excluyentes y hay pacientes que tienen más de un factor de riesgo.

Para evaluar la asociación de los signos clínicos con la edad se dicotomizó la muestra en relación a la edad en recién nacidos $(\mathrm{RN})$ y mayores de 28 días. A su vez, este último tramo de edad se dividió en menor a 6 años e igual o mayor de 6 años, considerando que el mayor desarrollo cerebral se produce antes de los 6 años. Los AVE se dividieron en isquémico arterial y trombosis de senos venosos ${ }^{23}$.

El análisis estadístico fue realizado usando SPSS. Las diferencias en las características demográficas (edad, sexo, presentación clínica) entre los grupos etarios fueron comparadas usando $\chi^{2}$ y Test exacto de Fisher para variables dicotómicas. Se establece un valor $<0,05$ para tener significado estadístico. Para cuantificar el grado de asociación se realizó OR. Para la edad se calculó mediana y el intervalo intercuartil, ya que los datos no presentaron distribución normal.

\section{Resultados}

Se reclutaron un total de 156 niños con AVE isquémico, 50 recién nacidos (RN) y 106 mayores de 28 días. Se excluye un caso con crisis isquémica transitoria y otro caso con datos no recuperables de ficha clínica. El promedio de edad y mediana al momento del diagnóstico, para la totalidad de los pacientes fue de $36 \mathrm{y}$ 4,9 meses respectivamente (rango intercuartil 5,8 días a 48 meses) y la proporción masculino/femenino fue de 1,7/1. El 67\% de los casos debutaron con signos neurológicos difusos y el $61 \%$ con convulsiones. Los signos neurológicos focales se encontraron sólo en el 33\% de los casos.

En relación al tipo de AVE, el 86\% correspondió a infarto arterial, el que se presentó a una edad mayor que la trombosis de senos venosos (mediana 5,04 meses vs 1,32 meses). No hubo diferencias en la presentación clínica según tipo de AVE.

Al dicotomizar la muestra en recién nacidos y mayores de 28 días, se obtuvieron diferencias estadísticamente significativas entre la presentación clínica de los recién nacidos y el grupo de mayor de 28 días, con un predominio de signos difusos $(\mathrm{p}=0,0001$ y OR $=47,2)$ y convulsiones $(p=0,0001$ y $O R=5,1)$ en $R N$ con signos focales infrecuentes $(\mathrm{p}=0,0001$ y $\mathrm{OR}=0,002)($ tabla 1$)$. No hubo diferencias significativas entre la presentación clínica y tipo de AVE (tabla 2).

Los signos difusos (compromiso de conciencia, trastornos de alimentación y apneas) 
se presentaron en 49/50 RN; sólo en 1 RN se pudo evidenciar un signo focal (hemiparesia)

Al estratificar la muestra de los mayores de 28 días en $<$ de 6 años y $\geq$ a 6 años, se observaron diferencias entre ambos grupos en relación al tipo de AVE. En cuanto a presentación clínica en menores de 6 años, predominaron los signos difusos $(\mathrm{p}=0,001$ y $\mathrm{OR}=18,6$; $\mathrm{IC}=6.9-50)$ y las convulsiones $(\mathrm{p}=0,01 \mathrm{y}$ $\mathrm{OR}=3,6 ; \mathrm{IC}=1,6-8,1)$ al igual que en recién nacidos. En la población mayor de 6 años (33 niños) predominaron en forma estadística- mente significativa los signos focales $(81,8 \%$ $\mathrm{p}=0,001)($ tabla 3$)$.

Los factores de riesgo más frecuentes para ambos tipo de AVE fueron la condición sistémica aguda, que acontece para el 55,8\% de todos los casos y es mayor para trombosis de senos venosos, pero sin significancia estadística). Le sigue en frecuencia las cardiopatías con un $32,7 \%$, donde predominan las cardiopatías congénitas $(28,2 \%)$ vs adquiridas $(4,5 \%)$. Las enfermedades crónicas y las enfermedades agudas de cabeza y cuello tiene una distribu-

Tabla 1. Características de 156 pacientes con AVE pediátrico

\begin{tabular}{|c|c|c|c|c|}
\hline & $\begin{array}{c}\text { Recién nacidos } \\
(n=50)\end{array}$ & $\begin{array}{c}>28 \text { días-18 años } \\
(n=106)\end{array}$ & Valor p & OR (IC) \\
\hline Media & 3,94 días & 4,3 años & & \\
\hline Mediana & 1,99 días & 2 años & & \\
\hline Rango intercuartil & 1-6 días & 4,9 mes- 7,8 años & & \\
\hline Sexo masculino & $34(68 \%)$ & $66(62,3 \%)$ & 0,3 & $1,3 \quad(0,6-2,6)$ \\
\hline Signos difusos & 49 (98\%) & $54(51 \%)$ & 0,0001 & $47,2 \quad(6,2-354,3)$ \\
\hline Convulsiones & $42(82 \%)$ & $54(51 \%)$ & 0,0001 & $5,1 \quad(2,2-11,8)$ \\
\hline Signos focales & $1 \quad(2 \%)$ & $52(49 \%)$ & 0,0001 & $0,02(0,003-0,16)$ \\
\hline AVE isquemico arterial & $41(82 \%)$ & $93(88 \%)$ & 0,16 & $0,7 \quad(0,43-1,3)$ \\
\hline trombosis de senos venosos & $9(18 \%)$ & $13(12 \%)$ & & \\
\hline
\end{tabular}

Tabla 2. Características clínicas de 156 niños según tipo de AVE

\begin{tabular}{|c|c|c|c|c|c|c|}
\hline & & Infarto arterial & TSV & valor $p$ & OR & (IC) \\
\hline n (156) & & 134 & 22 & & & \\
\hline Mediana edad & (años) & 0,42 & 0,11 & & & \\
\hline Neonatos & n $(\%)$ & $45(33,6)$ & $11(50)$ & $0,05^{*}$ & 0,51 & $(0,20-1,25)$ \\
\hline Sexo masculino & n $\quad(\%)$ & $85(63,4)$ & $15(68,2)$ & 0,6 & 0,9 & $(0,31-2,1)$ \\
\hline Signos difusos & n $(\%)$ & $89(66,4)$ & $15(68,2)$ & 0,6 & 0,9 & $(0,35-2,4)$ \\
\hline Convulsiones & n $\quad(\%)$ & $83(62)$ & $12(54,5)$ & 0,33 & 1,3 & $(0,55-3,56)$ \\
\hline Signos focales & n $(\%)$ & $45(33,5)$ & $7(31,8)$ & 0,5 & 1,8 & $(0,41-2,85)$ \\
\hline
\end{tabular}

Tabla 3. Características de 106 niños con AVE isquémico según estrato de edad

\begin{tabular}{|lcccc|}
\hline & $\begin{array}{c}<\mathbf{6} \text { años } \\
\mathbf{n = 7 3}\end{array}$ & $\begin{array}{c}>\mathbf{0} \text { igual } \mathbf{6} \text { años } \\
\mathbf{n = 3 3}\end{array}$ & valor $\mathbf{p}$ & OR (IC) \\
\hline Sexo masculino & $43(58,9)$ & $23(69,7)$ & 0,15 & $0,6(0,26 ; 1,5)$ \\
Signos difusos & $48(65,7)$ & $6(18,2)$ & 0,0001 & $8,6(3,2 ; 23,7)$ \\
Signos focales & $25(34,2)$ & $27(81,8)$ & 0,0001 & $0,1(0,04 ; 0,3)$ \\
Convulsiones & $42(57,5)$ & $12(36,4 \%)$ & 0,03 & $4,4(1,3-14,5)$ \\
AVE isquemico arterial & $68(93,2 \%)$ & $25(75,8 \%)$ & 0,016 & $0,2(0,07-0,8)$ \\
\hline Trombosis senos venosos & $5(6,8)$ & $8(24,2)$ & 0,016 & \\
\hline
\end{tabular}


Tabla 4. Factores de riesgo de AVE isquémico en 156 pacientes pediátricos

\begin{tabular}{|c|c|c|c|c|c|c|c|}
\hline \multirow[b]{2}{*}{ Factor de riesgo } & \multicolumn{2}{|c|}{ AVE arterial } & \multicolumn{2}{|c|}{$\begin{array}{l}\text { Trombosis senos } \\
\text { venosos }\end{array}$} & \multicolumn{2}{|c|}{ Total } & \multirow[t]{2}{*}{ valor $p$} \\
\hline & $\mathbf{n}$ & $\%$ & $\mathbf{n}$ & $\%$ & n & $\%$ & \\
\hline Enfermedad aguda sistémica & 71 & $(53,0)$ & 16 & $(72,7)$ & 87 & $(55,8)$ & 0,06 \\
\hline Cardiopatías (congénitas y adquiridas) & 45 & $(33,6)$ & 6 & $(27,3)$ & 51 & $(32,7)$ & 0,55 \\
\hline Enfermedad crónica subyacente & 35 & $(22,4)$ & 6 & $(27,3)$ & 41 & $(26,3)$ & 0,9 \\
\hline Enfermedad aguda de cabeza y cuello & 31 & $(23,1)$ & 7 & $(31,8)$ & 38 & $(24,4)$ & 0,37 \\
\hline Vasculopatías & 14 & $(10,4)$ & 3 & $(13,6)$ & 17 & $(10,9)$ & 0,6 \\
\hline
\end{tabular}

ción similar y las vasculopatías son las menos frecuentes $(10,9 \%)$ (tabla 4$)$.

\section{Discusión}

En nuestro estudio, la presentación clínica del AVE pediátrico, tuvo como síntomas cardinales el compromiso difuso del sistema nervioso central y las convulsiones. Ambos síntomas van disminuyendo en frecuencia a medida que aumenta la edad. A mayor edad, los niños ya comienzan a focalizar las lesiones cerebrales y después de los 6 años, el porcentaje de signos focales como presentación inicial de un AVE fue de $81,8 \%$ y las convulsiones disminuyeron desde el $82 \%$ para los recién nacidos a $36 \%$ en población de escolares mayor de 6 años. En la población adulta, en cambio, predominan los signos focales y las convulsiones son infrecuentes en las primeras $24 \mathrm{~h}$ del evento ${ }^{24}$.

Comparando nuestras cifras con los reportes de Singh et $\mathrm{al}^{25} \mathrm{y}$ Abend et $\mathrm{al}^{18}$, en cuanto a convulsiones como síntoma de presentación inicial, tenemos diferencias importantes ya que en nuestra serie tenemos un $61 \%$ de convulsiones como presentación inicial en la muestra de 156 niños y ellos reportan un 21 y $22 \%$ respectivamente. Creemos que la diferencia radica en que en ambos casos excluyeron recién nacidos (33\% de nuestra población), que el promedio de edad de los niños es mayor (8,4 y 5,7 años vs 3 años del nuestro) y que el tamaño muestral es inferior ( 77 y 60 casos respectivamente).

En relación a la presentación inicial con signos focales, Srinivasan ${ }^{26}$, presenta 107 niños con AVE isquémico (82 mayores de 28 días) e informa un porcentaje alto de signos focales, $(86 \%)$ en relación al 51\% nuestro. Esta diferencia puede ser porque Srinivisan Incluye como signo focal convulsiones focales que en nuestro trabajo están incluidos como convulsiones. No informa el promedio de edad de su muestra, por lo que no podemos saber si el $20 \%$ de déficit visual, afasia o trastornos sensoriales fueron encontrados en población mayor, que puede definir su síntoma. En nuestro trabajo, la población mayor de 6 años también presenta un alto porcentaje de déficit focal $(81,8 \%)$ que desaparece en la población de menor edad. Hartman et $\mathrm{al}^{27}$, presenta 125 pacientes con AVE y describe compromiso de conciencia, cefalea y convulsiones en el 67 , 100 y $83 \%$ de los casos en las primeras $24 \mathrm{~h}$ del evento, pero en su muestra igualmente excluye recién nacidos e incluye AVE hemorrágicos, los que forman el $50 \%$ de su muestra.

En cuanto a los factores de riesgo, en el $83,5 \%$ de los casos, se encontró un factor de riesgo. El más frecuente fue la enfermedad sistémica aguda seguida de cardiopatías y enfermedades crónicas. Es importante señalar que en 23 pacientes $(14,7 \%)$ no hubo factor de riesgo identificable. Estos resultados difieren de lo encontrado por Mackay ${ }^{22}$, quien reportó que la condición más frecuentemente encontrada en una muestra de 676 niños fue la arteriopatía con un $53 \%$, seguida de las cardiopatías. Al igual que los trabajos anteriores, excluye recién nacidos, lo que la hace diferente de nuestra muestra y que en el $80 \%$ de los casos tenían además estudio vascular con angioresonancia angioTAC o angiografía convencional, lo que no sucedió y es una falencia en nuestro estudio ya que es necesario para la prevención 
secundaria. El conocer si el AVE arterial incluye una estenosis arterial tiene importantes implicancias, por cuanto el riesgo de recurrencias en estos casos es tan alto como $66 \%$ dentro de los primeros 5 años ${ }^{5,28}$.

La fortaleza de este trabajo radica en que es el primer estudio en Chile de AVE infantil, la población es numerosa comparada con otras series, el seguimiento fue prospectivo y se obtuvieron imágenes cerebrales en el 100\% de los casos. Entre las debilidades de nuestro trabajo está el hecho que la recolección de los datos es tardía porque el diagnóstico es tardío y probablemente la información de las primeras 24-48 h pudiera estar sesgada.

Es importante realizar esfuerzos para aumentar la sospecha de AVE, tanto en la población general como en el equipo médico, considerando que la mortalidad es de 12 a $15 \%$, la morbilidad es de $60-70 \%$ y hay hasta un $35 \%$ de recurrencias en niños mayores de 28 días $^{5}$. Dada la poca especificidad de la presentación clínica encontrada, pensamos que hay una necesidad de instituir medidas de prevención, diagnóstico precoz cuidado especiales de AVE y prevención de recurrencias.

\section{Referencias}

1.- World Health Organization: Cerebrovascular Disorders. 1998; Available at: whqlibdoc.who.int/offset/ WHO_OFFSET_43.pd. Accessed Junio, 2012.

2.- Saver JL: Time is brain-quantified. Stroke 2006; 37 (1): 263-6.

3.- Muresan I, Favrole P, Levy P, Andreux F, Marro B, Alamowitch $S$ : Very early neurologic improvement after intravenous thrombolysis. Arch Neurol 2010; 67 (11): 1323-8.

4.- Kerr LM, Anderson DM, Thompson JA, Lyver SM, Call $G K$ : Ischemic stroke in the young: evaluation and age comparison of patients six months to thirty-nine years. J Child Neurol 1993; 8 (3): 266-70.

5.- Ganesan V, Hogan A, Shack N, Gordon A, Isaacs E, Kirkham FJ: Outcome after ischaemic stroke in childhood. Dev Med Child Neurol 2000; 42 (7): 455-61.

6.- Fullerton HJ, Wu YW, Zhao S, Johnston SC: Risk of stroke in children: ethnic and gender disparities. Neurology 2003; 61 (2): 189-94.

7.- Amlie-Lefond C, Chan AKC, Kirton A, et al: Thrombolysis in acute childhood stroke: Design and challenges of the thrombolysis in pediatric stroke clinical trial. Neuroepidemiology 2009; 32 (4): 279-86.

8.- Amlie-Lefond C, deVeber G, Chan AK, et al: Use of alteplase in childhood arterial ischaemic stroke: a multicentre, observational, cohort study. Lancet Neurol 2009; 8 (6): 530-6.

9.- Ciccone S, Cappella M, Borgna-Pignatti C: Ischemic stroke in infants and children: Practical management in emergency. Stroke Research and Treatment 2011.

10.- Carpenter J, Tsuchida T, Lynch JK: Treatment of arterial ischemic stroke in children. Expert Rev Neurother 2007; 7 (4): 383-92.

11.- Braun KP, Kappelle LJ, Kirkham FJ, Deveber G: Diagnostic pitfalls in paediatric ischaemic stroke. Dev Med Child Neurol 2006; 48 (12): 985-90.

12.- deVeber $G A$ : Delays in the timely diagnosis of stroke in children. Nat Rev Neurol 2010; 6 (2): 64-6.

13.- Gabis LV, Yangala R, Lenn NJ: Time lag to diagnosis of stroke in children. Pediatrics 2002; 110 (5): 924-8.

14.- Bernard TJ, Fenton LZ, Apkon SD, et al: Biomarkers of Hypercoagulability and Inflammation in ChildhoodOnset Arterial Ischemic Stroke. J Pediatr 2010; 156 (4): 651-6.

15.- Bernard TJ, Manco-Johnson MJ, Goldenberg NA: The roles of anatomic factors, thrombophilia, and antithrombotic therapies in childhood-onset arterial ischemic stroke. Thromb Res 2011; 127 (1): 6-12.

16.- Barreirinho $S$, Ferro A, Santos $M$, et al: Inherited and acquired risk factors and their combined effects in pediatric stroke. Pediatr Neurol 2003; 28 (2): 134-8.

17.- Mackay MT, Wiznitzer M, Benedict SL, et al: Arterial ischemic stroke risk factors: the International Pediatric Stroke Study. Ann Neurol 2011; 69 (1): 130-40.

18.- Abend NS, Beslow LA, Smith SE, et al: Seizures as a presenting symptom of acute arterial ischemic stroke in childhood. J Pediatr 2011; 159 (3): 479-83.

19.- deVeber $G$ : Arterial ischemic strokes in infants and children: an overview of current approaches. Semin Thromb Hemost 2003; 29 (6): 567-73.

20.- Kirton A, Armstrong-Wells J, Chang T, et al: Symptomatic neonatal arterial ischemic stroke: the International Pediatric Stroke Study. Pediatrics 2011; 128 (6): e140210.

21.- Wusthoff CJ, Kessler SK, Vossough A, et al: Risk of later seizure after perinatal arterial ischemic stroke: A prospective cohort study. Pediatrics 2011; 127 (6): e1550-e7.

22.- MacKay MT, Wiznitzer M, Benedict SL, Lee KJ, Deveber GA, Ganesan $V$ : Arterial ischemic stroke risk factors: The international pediatric stroke study. Ann Neurol 2011; 69 (1): 130-40. 
23.- Dlamini N, Kirkham FJ: Stroke and cerebrovascular disorders. Curr Opin Pediatr 2009; 21 (6): 751-61.

24.- Szaflarski JP, Rackley AY, Kleindorfer DO, et al: Incidence of seizures in the acute phase of stroke: A population-based study. Epilepsia (Series 4) 2008; 49 (6): 974-81.

25.- Singh RK, Zecavati $N$, Singh J, et al: Seizures in acute childhood stroke. J Pediatr 2012; 160 (2): 291-6.

26.- Srinivasan J, Miller SP, Phan TG, Mackay MT: Delayed recognition of initial stroke in children: need for increased awareness. Pediatrics 2009; 124 (2): e227-34.

27.- Hartman AL, Lunney KM, Serena JE: Pediatric Stroke: Do Clinical Factors Predict Delays in Presentation? J Pediatr 2009; 154 (5): 727-32.e1.

28.- Fullerton HJ, Wu YW, Sidney S, Johnston SC: Risk of recurrent childhood arterial ischemic stroke in a population-based cohort: the importance of cerebrovascular imaging. Pediatrics 2007; 119 (3): 495-501. 